\title{
Comparison of Integral-Equation Formulations for the Fast and Accurate Solution of Scattering Problems Involving Dielectric Objects with the Multilevel Fast Multipole Algorithm
}

\author{
Özgür Ergül, Student Member, IEEE, and Levent Gürel, Fellow, IEEE
}

\begin{abstract}
We consider fast and accurate solutions of scattering problems involving increasingly large dielectric objects formulated by surface integral equations. We compare various formulations when the objects are discretized with Rao-Wilton-Glisson functions, and the resulting matrix equations are solved iteratively by employing the multilevel fast multipole algorithm (MLFMA). For large problems, we show that a combined-field formulation, namely, the electric and magnetic current combined-field integral equation (JMCFIE), requires fewer iterations than other formulations within the context of MLFMA. In addition to its efficiency, JMCFIE is also more accurate than the normal formulations and becomes preferable, especially when the problems cannot be solved easily with the tangential formulations.
\end{abstract}

Index Terms-Dielectrics, iterative solutions, multilevel fast multipole algorithm (MLFMA), surface integral equations.

\section{INTRODUCTION}

$\mathbf{S}$ URFACE integral equations (SIE) are commonly used to formulate electromagnetic scattering problems involving three-dimensional dielectric objects with arbitrary shapes [1]. Using the equivalence principle, electric and magnetic currents are defined on the surface of the scatterer. By means of a simultaneous discretization of the geometry and the integral equations, equivalent surface currents are expanded in a series of basis functions. Then, the coefficients of the basis functions are calculated by solving the dense matrix equations, which are obtained by testing the boundary conditions for the electric and magnetic fields on the surface of the object [2]. Many dielectric formulations are derived by using various combinations of the boundary conditions, testing schemes, and scaling operations [3]-[16]. Some of these methods are known to be stable and provide accurate results, although their performances may vary significantly in terms of efficiency and accuracy.

\footnotetext{
Manuscript received January 07, 2008; revised August 31, 2008. Current version published March 04, 2009. This work was supported in part by the Scientific and Technical Research Council of Turkey (TUBITAK) under Research Grant 105E172, in part by the Turkish Academy of Sciences in the framework of the Young Scientist Award Program (LG/TUBA-GEBIP/2002-1-12), and in part by contracts from ASELSAN and SSM.

The authors are with the Department of Electrical and Electronics Engineering, Bilkent University, TR-06800 Bilkent, Ankara, Turkey and also with the Computational Electromagnetics Research Center (BiLCEM), Bilkent University, TR-06800 Bilkent, Ankara, Turkey (e-mail: ergul@ee.bilkent.edu.tr; lgurel@bilkent.edu.tr).

Digital Object Identifier 10.1109/TAP.2008.2009665
}

For homogeneous dielectric objects, surface formulations are generally constructed by combining tangential (T) and/or normal $(\mathrm{N})$ equations. In the $\mathrm{T}$ equations, boundary conditions are tested directly by sampling the tangential components of the fields on the surface. In the $\mathrm{N}$ equations, however, fields are tested after they are projected onto the surface via a cross-product operation with the outward normal vector. In both cases, we assume a Galerkin scheme using the same set of functions to expand the current densities (basis functions) and to test the boundary conditions (testing functions). Considering the boundary conditions for the electric and magnetic fields separately, we can derive four different integral equations, namely, the tangential electric-field integral equation (T-EFIE), the normal electric-field integral equation (N-EFIE), the tangential magnetic-field integral equation (T-MFIE), and the normal magnetic-field integral equation (N-MFIE) [9]. These equations are derived for both the inner and outer regions and can be combined in various ways. To avoid numerical internal resonances, it is preferable to derive a combined-field integral equation (CFIE) by linearly combining EFIE and MFIE [8]. For example, a combination of T-EFIE and T-MFIE leads to a T-T-CFIE formulation. Similarly, one can obtain T-N-CFIE (T-EFIE+N-MFIE), N-T-CFIE (N-EFIE+T-MFIE), and N-N-CFIE (N-EFIE+N-MFIE) by combining EFIE and MFIE appropriately. In these formulations, equations obtained for the inner and outer regions are solved simultaneously to obtain the two sets of unknowns, i.e., electric and magnetic currents. We can also derive various other formulations involving triple combinations, such as TN-N-CFIE (T-EFIE+N-EFIE+N-MFIE), for more stable solutions [9].

CFIE formulations described above are based on a linear combination of EFIE and MFIE in the same way for each medium, while it is also possible to use different combinations for the inner and outer regions [10]. Alternatively, we can linearly combine the inner and outer equations while solving EFIE and MFIE simultaneously. In this class of formulations, Poggio-Miller-Chang-Harrington-Wu-Tsai (PMCHWT) [1], [4], [5] and Müller [3] formulations are well known and commonly used in the literature. Both $\mathrm{T}$ and $\mathrm{N}$ versions of these formulations are possible, but only the tangential PMCHWT (T-PMCHWT) formulation and the normal Müller formulation (NMF) are stable, whereas the normal PMCHWT and the tangential Müller formulations are usually unstable [11], [13]. Recently, remarkable efforts have been made to further improve 
the dielectric formulations by devising novel combinations of the integral equations. For example, the combined tangential formulation (CTF) is similar to the T-PMCHWT formulation, but it involves a careful (and improved) scaling of T-EFIE and T-MFIE [14]. A similar combination of N-EFIE and N-MFIE leads to the combined normal formulation (CNF) [14]. Finally, the modified NMF (MNMF) is obtained by normalizing the equations in NMF to produce better-conditioned matrix equations [13].

In this paper, we consider the iterative solution of relatively large dielectric problems using the multilevel fast multipole algorithm (MLFMA) [17]. We compare both the accuracy and efficiency of the solutions obtained by using different formulations. Specifically, we consider CTF, CNF, MNMF, and a formulation called the electric and magnetic current combined-field integral equation (JMCFIE) [12], which is derived by combining $\mathrm{CTF}$ and CNF. These formulations have recently been investigated for the solution of scattering problems involving relatively small objects [14]. However, one needs to compare them also when the problem size is large and the solution is performed iteratively. In general, our results show that JMCFIE becomes the most efficient formulation for large problems since it requires fewer iterations than other formulations. In addition, scattered fields obtained by JMCFIE are more accurate than those obtained by the $\mathrm{N}$ formulations, such as $\mathrm{CNF}$ and MNMF.

This paper is organized as follows. In the next section, we summarize surface formulations of dielectric problems. Section III presents accurate and robust discretizations of the surface integral equations. Then, Section IV discusses how to calculate the far-field interactions efficiently using MLFMA, and Section $\mathrm{V}$ presents block-diagonal preconditioning to accelerate the convergence of iterative solutions. Finally, numerical results are presented in Section VI, followed by our concluding remarks in Section VII.

\section{Surface Formulations of Dielectric Problems}

To derive the surface integral equations, operators for the outside $(l=1)$ and inside $(l=2)$ the object are defined as

$$
\begin{aligned}
& \mathcal{T}_{l}\{\boldsymbol{X}\}(\boldsymbol{r})=i k_{l} \int_{S} d \boldsymbol{r}^{\prime}\left[\boldsymbol{X}\left(\boldsymbol{r}^{\prime}\right)+\frac{1}{k_{l}^{2}} \nabla^{\prime} \cdot \boldsymbol{X}\left(\boldsymbol{r}^{\prime}\right) \nabla\right] g_{l}\left(\boldsymbol{r}, \boldsymbol{r}^{\prime}\right) \\
& \mathcal{K}_{l}\{\boldsymbol{X}\}(\boldsymbol{r})=\int_{\mathrm{PV}, S} d \boldsymbol{r}^{\prime} \boldsymbol{X}\left(\boldsymbol{r}^{\prime}\right) \times \nabla^{\prime} g_{l}\left(\boldsymbol{r}, \boldsymbol{r}^{\prime}\right)
\end{aligned}
$$

where PV indicates the principal value of the integral, $\boldsymbol{X}(\boldsymbol{r})$ is either the equivalent electric current $\boldsymbol{J}(\boldsymbol{r})$ or the equivalent magnetic current $\boldsymbol{M}(\boldsymbol{r})$ on the surface of the object $S, k_{l}$ is the wavenumber associated with medium $l$, and

$$
g_{l}\left(\boldsymbol{r}, \boldsymbol{r}^{\prime}\right)=\frac{\exp \left(i k_{l} R\right)}{4 \pi R} \quad\left(R=\left|\boldsymbol{r}-\boldsymbol{r}^{\prime}\right|\right)
$$

denotes the homogeneous-space Green's function. Using the operators in (1) and (2), the T formulations can be derived as [14]

$$
\begin{aligned}
& -\hat{\boldsymbol{t}} \cdot\left[a \eta_{1}^{-1}\left(\mathcal{K}_{1}+0.5 \mathcal{I}_{n}\right)+b \eta_{2}^{-1}\left(\mathcal{K}_{2}-0.5 \mathcal{I}_{n}\right)\right]\{\boldsymbol{M}\}(\boldsymbol{r}) \\
& \quad+\hat{\boldsymbol{t}} \cdot\left[a \mathcal{T}_{1}+b \mathcal{I}_{2}\right]\{\boldsymbol{J}\}(\boldsymbol{r})=-a \eta_{1}^{-1} \hat{\boldsymbol{t}} \cdot \boldsymbol{E}^{i}(\boldsymbol{r})
\end{aligned}
$$

$$
\begin{aligned}
\hat{\boldsymbol{t}} \cdot & {\left[c \eta_{1}\left(\mathcal{K}_{1}+0.5 \mathcal{I}_{n}\right)+d \eta_{2}\left(\mathcal{K}_{2}-0.5 \mathcal{I}_{n}\right)\right]\{\boldsymbol{J}\}(\boldsymbol{r}) } \\
& +\hat{\boldsymbol{t}} \cdot\left[c \mathcal{T}_{1}+d \mathcal{T}_{2}\right]\{\boldsymbol{M}\}(\boldsymbol{r})=-c \eta_{1} \hat{\boldsymbol{t}} \cdot \boldsymbol{H}^{i}(\boldsymbol{r})
\end{aligned}
$$

where $\boldsymbol{E}^{i}(\boldsymbol{r})$ and $\boldsymbol{H}^{i}(\boldsymbol{r})$ are the incident electric and magnetic fields, $\eta_{l}$ is the impedance of medium $l$, and $\hat{\boldsymbol{t}}$ is any tangential vector at the observation point $\boldsymbol{r}$ on the surface. In (4) and (5), $\mathcal{I}_{n}\{\boldsymbol{X}\}(\boldsymbol{r})=\hat{\boldsymbol{n}} \times \boldsymbol{X}(\boldsymbol{r})$, where $\hat{\boldsymbol{n}}$ is the outward normal vector at the observation point. Among infinitely many possibilities for the scalars $\{a, b, c, d\}$, several choices provide stable formulations, such as

$$
a=\eta_{1}, b=\eta_{2}, c=1 / \eta_{1}, d=1 / \eta_{2}
$$

and

$$
a=b=c=d=1
$$

which lead to the T-PMCHWT formulation [1], [4], [5] and CTF [14], respectively. Matrix equations obtained from CTF have identical diagonal partitions ${ }^{1}$, and they are usually better conditioned than the matrix equations obtained from the T-PMCHWT formulation [14].

Different from the $\mathrm{T}$ formulations, the $\mathrm{N}$ formulations are derived as

$$
\begin{aligned}
& \hat{\boldsymbol{n}} \times {\left[a\left(\mathcal{K}_{1}+0.5 \mathcal{I}_{n}\right)-b\left(\mathcal{K}_{2}-0.5 \mathcal{I}_{n}\right)\right]\{\boldsymbol{J}\}(\boldsymbol{r}) } \\
&+\hat{\boldsymbol{n}} \times\left[a \eta_{1}^{-1} \mathcal{T}_{1}-b \eta_{2}^{-1} \mathcal{T}_{2}\right]\{\boldsymbol{M}\}(\boldsymbol{r})=-a \hat{\boldsymbol{n}} \times \boldsymbol{H}^{i}(\boldsymbol{r}) \\
& \hat{\boldsymbol{n}} \times {\left[c\left(\mathcal{K}_{1}+0.5 \mathcal{I}_{n}\right)-d\left(\mathcal{K}_{2}-0.5 \mathcal{I}_{n}\right)\right]\{\boldsymbol{M}\}(\boldsymbol{r}) } \\
& \quad+\hat{\boldsymbol{n}} \times\left[-c \eta_{1} \mathcal{T}_{1}+d \eta_{2} \mathcal{T}_{2}\right]\{\boldsymbol{J}\}(\boldsymbol{r})=c \hat{\boldsymbol{n}} \times \boldsymbol{E}^{i}(\boldsymbol{r})
\end{aligned}
$$

where the choices

$$
a=\mu_{1}, b=\mu_{2}, c=\epsilon_{1}, d=\epsilon_{2}
$$

and

$$
a=b=c=d=1
$$

lead to NMF [13] and CNF [14], respectively. Using a Galerkin scheme, matrix equations obtained by using the $\mathrm{N}$ formulations are usually better conditioned than those obtained with the $\mathrm{T}$ formulations. This is because the $\mathrm{N}$ formulations involve welltested identity operators, which appear on the diagonal partitions of the matrix equations. Finally, choosing the scalars as

$$
\begin{aligned}
& a=\mu_{1} /\left(\mu_{1}+\mu_{2}\right), b=\mu_{2} /\left(\mu_{1}+\mu_{2}\right), \\
& c=\epsilon_{1} /\left(\epsilon_{1}+\epsilon_{2}\right), d=\epsilon_{2} /\left(\epsilon_{1}+\epsilon_{2}\right)
\end{aligned}
$$

in (8) leads to MNMF, which usually produces better-conditioned matrix equations than NMF [13].

When the scatterer is a perfect electric conductor (PEC), the operators associated with the "inner" medium $(l=2)$ and the magnetic current disappear in the $\mathrm{T}$ and $\mathrm{N}$ formulations in (4)-(5) and (8)-(9). Then, T-EFIE and T-MFIE in the T formulations are decoupled, and they can be solved independently to obtain the induced electric current on the surface of the object. Although T-MFIE is extremely unstable, T-EFIE is stable and is commonly used in the literature [19]. Similarly, in the $\mathrm{N}$ formulations, N-MFIE (stable) and N-EFIE (unstable) are decoupled for PEC objects. Finally, we also consider a linear combination of CTF and CNF, which is denoted as JMCFIE [12], for dielectric objects. We note that JMCFIE reduces to two forms of

\footnotetext{
${ }^{1}$ Discretization of dielectric formulations leads to matrix equations with $2 \times 2$ partitions as detailed in Section III
} 
CFIE for PEC objects, while only one form involving T-EFIE and N-MFIE is stable.

In this paper, we compare CTF, CNF, MNMF, and JMCFIE for the solution of scattering problems involving only homogeneous dielectric objects. However, these formulations can easily be extended to those problems including composite structures with multiple dielectric and PEC regions. The generalized procedure consists of the following main stages.

- Formulate an equivalent problem for each non-PEC domain by defining equivalent currents and applying the boundary conditions on the surfaces.

- Perform the discretization process detailed in Section III for each domain by employing oriented basis and testing functions.

- Combine the related unknowns on the boundaries and the corresponding equations to form a single matrix equation to solve.

This generalized procedure is extensively discussed in [18] in the context of a EFIE-CFIE-PMCHWT formulation, together with various techniques to handle the junctions, i.e., those locations where three or more domains intersect.

\section{DISCRETIZATION OF THE INTEGRAL EQUATIONS}

For the numerical solution of surface integral equations, we discretize the surfaces by using small planar triangles and employ Rao-Wilton-Glisson (RWG) [19] basis functions to expand the unknown surface current densities. Using a Galerkin scheme, we also choose the testing functions as RWG. Then, the interaction between the $m$ th testing function $\boldsymbol{t}_{m}(\boldsymbol{r})$ and the $n$th basis function $\boldsymbol{b}_{n}(\boldsymbol{r})$ are defined for different operators $(\mathcal{K}$ and $\mathcal{T}$ ) and equation types ( $\mathrm{T}$ or $\mathrm{N}$ ) as

$$
\begin{aligned}
K_{l}^{T}[m, n]= & \int_{S_{m}} d \boldsymbol{r} \boldsymbol{t}_{m}(\boldsymbol{r}) \cdot \int_{\mathrm{PV}, S_{n}} d \boldsymbol{r}^{\prime} \boldsymbol{b}_{n}\left(\boldsymbol{r}^{\prime}\right) \times \nabla^{\prime} g_{l}\left(\boldsymbol{r}, \boldsymbol{r}^{\prime}\right) \\
K_{l}^{N}[m, n]= & \int_{S_{m}} d \boldsymbol{r} \boldsymbol{t}_{m}(\boldsymbol{r}) \cdot \hat{\boldsymbol{n}} \int_{\mathrm{PV}, S_{n}} d \boldsymbol{r}^{\prime} \boldsymbol{b}_{n}\left(\boldsymbol{r}^{\prime}\right) \times \nabla^{\prime} g_{l}\left(\boldsymbol{r}, \boldsymbol{r}^{\prime}\right) \\
T_{l}^{T}[m, n]= & i k_{l} \int_{S_{m}} d \boldsymbol{r} \boldsymbol{t}_{m}(\boldsymbol{r}) \cdot \int_{S_{n}} d \boldsymbol{r}^{\prime} \boldsymbol{b}_{n}\left(\boldsymbol{r}^{\prime}\right) g_{l}\left(\boldsymbol{r}, \boldsymbol{r}^{\prime}\right) \\
& -\frac{i}{k_{l}} \int_{S_{m}} d \boldsymbol{r} \boldsymbol{r}_{m}(\boldsymbol{r}) \cdot \int_{S_{n}} d \boldsymbol{r}^{\prime} \nabla^{\prime} \cdot \boldsymbol{b}_{n}\left(\boldsymbol{r}^{\prime}\right) \nabla^{\prime} g_{l}\left(\boldsymbol{r}, \boldsymbol{r}^{\prime}\right) \\
T_{l}^{N}[m, n]= & i k_{l} \int_{S_{m}} d \boldsymbol{r} \boldsymbol{t}_{m}(\boldsymbol{r}) \cdot \hat{\boldsymbol{n}} \times \int_{S_{n}} d \boldsymbol{r}^{\prime} \boldsymbol{b}_{n}\left(\boldsymbol{r}^{\prime}\right) g_{l}\left(\boldsymbol{r}, \boldsymbol{r}^{\prime}\right) \\
& -\frac{i}{k_{l}} \int_{S_{m}} d \boldsymbol{r} \boldsymbol{r}_{m}(\boldsymbol{r}) \cdot \hat{\boldsymbol{n}} \times \int_{S_{n}} d \boldsymbol{r}^{\prime} \nabla^{\prime} \cdot \boldsymbol{b}_{n}\left(\boldsymbol{r}^{\prime}\right) \nabla^{\prime} g_{l}\left(\boldsymbol{r}, \boldsymbol{r}^{\prime}\right)
\end{aligned}
$$

where $S_{m}$ and $S_{n}$ represent the spatial supports of $\boldsymbol{t}_{m}(\boldsymbol{r})$ and $\boldsymbol{b}_{n}(\boldsymbol{r})$, respectively. The interactions are calculated for $m, n=$ $1,2, \ldots, N$, where $N$ is the number of RWG functions used to expand the electric and magnetic currents. Accurate calculations of the integrals in (13)-(16) can be summarized as follows.

- Using RWG functions, the interactions in (13) and (14) can be modified as [20]

$$
K_{l}^{T}[m, n]=\int_{S_{m}} d \boldsymbol{r} \boldsymbol{r}_{m}(\boldsymbol{r}) \cdot \boldsymbol{b}_{n}(\boldsymbol{r}) \times \int_{\mathrm{PV}, S_{n}} d \boldsymbol{r}^{\prime} \nabla^{\prime} g_{l}\left(\boldsymbol{r}, \boldsymbol{r}^{\prime}\right)
$$

$K_{l}^{N}[m, n]=\int_{S_{m}} d \boldsymbol{r}\left(\boldsymbol{t}_{m}(\boldsymbol{r}) \times \hat{\boldsymbol{n}}\right) \cdot \boldsymbol{b}_{n}(\boldsymbol{r}) \times \int_{\mathrm{PV}, S_{n}} d \boldsymbol{r}^{\prime} \nabla^{\prime} g_{l}\left(\boldsymbol{r}, \boldsymbol{r}^{\prime}\right)$

where the outer integrals are evaluated numerically by employing a Gaussian quadrature rule [21]. Only the principle value of the inner integral is required, so that (17) and (18) are not evaluated for the self interactions of the triangles. However, the value of the inner integral is infinite when the testing point is on the edge of the source triangle. Since the singularity is logarithmic and it is quite mild, interaction of two near-neighboring (touching) triangles can be calculated accurately by sampling the observation points strictly inside the testing triangle. In addition, the accuracy and efficiency of the calculations can be improved by extracting the singularity, as detailed in [22].

- Using divergence-conforming RWG functions, the interaction in (15) is modified as

$$
\begin{array}{r}
T_{l}^{T}[m, n]=i k_{l} \int_{S_{m}} d \boldsymbol{r} \boldsymbol{t}_{m}(\boldsymbol{r}) \cdot \int_{S_{n}} d \boldsymbol{r}^{\prime} \boldsymbol{b}_{n}\left(\boldsymbol{r}^{\prime}\right) g_{l}\left(\boldsymbol{r}, \boldsymbol{r}^{\prime}\right) \\
-\frac{i}{k_{l}} \int_{S_{m}} d \boldsymbol{r} \nabla \cdot \boldsymbol{t}_{m}(\boldsymbol{r}) \int_{S_{n}} d \boldsymbol{r}^{\prime} \nabla^{\prime} \cdot \boldsymbol{b}_{n}\left(\boldsymbol{r}^{\prime}\right) g_{l}\left(\boldsymbol{r}, \boldsymbol{r}^{\prime}\right)
\end{array}
$$

by moving the differential operator onto the testing function in the second term. This is commonly practiced for T-EFIE formulations of PEC objects [19]. For the second term of (16), however, the differential operator is kept on the Green's function. Then, this term is calculated similarly to the interaction in (14). In contrast to (14), however, (16) should also be calculated for the self interactions of the triangles.

- The inner integrals in (13)-(16) are calculated as

$$
\begin{aligned}
I= & \int_{S_{n}} d \boldsymbol{r}^{\prime} f\left(\boldsymbol{r}^{\prime}\right) g_{l}\left(\boldsymbol{r}, \boldsymbol{r}^{\prime}\right) \\
= & \frac{1}{4 \pi} \int_{S_{n}} d \boldsymbol{r}^{\prime} f\left(\boldsymbol{r}^{\prime}\right) \frac{\exp \left(i k_{l} R\right)-1}{R} \\
& +\frac{1}{4 \pi} \int_{S_{n}} d \boldsymbol{r}^{\prime} f\left(\boldsymbol{r}^{\prime}\right) \frac{1}{R}=I_{1}+I_{2}
\end{aligned}
$$

and

$$
\begin{aligned}
\boldsymbol{I}= & \int_{S_{n}} d \boldsymbol{r}^{\prime} \nabla^{\prime} g_{l}\left(\boldsymbol{r}, \boldsymbol{r}^{\prime}\right) \\
= & \frac{1}{4 \pi} \int_{S_{n}} d \boldsymbol{r}^{\prime} \nabla^{\prime}\left(\frac{\exp \left(i k_{l} R\right)-1+0.5 k_{l}^{2} R^{2}}{R}\right) \\
& +\frac{1}{4 \pi} \int_{S_{n}} d \boldsymbol{r}^{\prime} \nabla^{\prime}\left(\frac{1}{R}\right)-\frac{k_{l}^{2}}{8 \pi} \int_{S_{n}} d \boldsymbol{r}^{\prime} \nabla^{\prime} R \\
= & \boldsymbol{I}_{1}+\boldsymbol{I}_{2}+\boldsymbol{I}_{3}
\end{aligned}
$$

where $f\left(\boldsymbol{r}^{\prime}\right)=\left\{1, x^{\prime}, y^{\prime}\right\}$ in (20). We perform coordinate transformations for efficiency [23]. In (20) and (21), we note that

$$
\begin{aligned}
& \lim _{R \rightarrow 0} \frac{\exp \left(i k_{l} R\right)-1}{R}=i k_{l} \\
& \lim _{R \rightarrow 0} \nabla^{\prime}\left(\frac{\exp \left(i k_{l} R\right)-1+0.5 k_{l}^{2} R^{2}}{R}\right)=0 .
\end{aligned}
$$

Therefore, $I_{1}$ and $\boldsymbol{I}_{1}$ can be calculated numerically using an adaptive integration method [23] or a Gaussian quadra- 
ture rule. The remaining terms, i.e., $I_{2}, \boldsymbol{I}_{2}$, and $\boldsymbol{I}_{3}$ are evaluated analytically [20], [24], [25].

The processing time for the calculation of the interactions in (13)-(16) depends on the medium parameters. When the relative permittivity or permeability of a medium increases, it becomes difficult to evaluate the interactions since the integrands become more oscillatory. In such cases, accurate calculations of the interactions require extracting more terms to smooth the integrands or increasing the number of sampling points for the numerical integrations.

Calculating the interactions in (13)-(16), $2 N \times 2 N$ matrix equations are constructed as

$$
\left[\begin{array}{ll}
\bar{Z}_{11} & \overline{\boldsymbol{Z}}_{12} \\
\overline{\boldsymbol{Z}}_{21} & \overline{\boldsymbol{Z}}_{22}
\end{array}\right] \cdot\left[\begin{array}{c}
\boldsymbol{a}_{J} \\
\boldsymbol{a}_{M}
\end{array}\right]=\left[\begin{array}{c}
\boldsymbol{v}_{1}^{\text {inc }} \\
\boldsymbol{v}_{2}^{\text {inc }}
\end{array}\right]
$$

where $\boldsymbol{a}_{J}$ and $\boldsymbol{a}_{M}$ are the coefficients of the RWG functions expanding the electric and magnetic currents, respectively, and $\boldsymbol{v}_{1,2}^{\text {inc }}$ represents the excitation vectors obtained by testing the incident fields. Upper partitions of the $2 N \times 2 N$ matrix, i.e., $\bar{Z}_{11}$ and $\bar{Z}_{12}$, are obtained by the discretization of (4) or (8), or their combination, depending on the type of the dielectric formulation. Similarly, lower partitions $\bar{Z}_{21}$ and $\bar{Z}_{22}$ are obtained from (5) or (9), or their combination.

In MLFMA, there are $O(N)$ near-field interactions, which are calculated directly during the initial setup stage of the program and stored in memory, to be used multiple times during the iterations. When the problem size is large, the setup of MLFMA usually requires negligible time, compared to the iterative solution part. Therefore, the long setup time of JMCFIE, compared with CTF, CNF, and MNMF, is negligible when the overall time is considered for large problems. However, near-field interactions require a significant amount of memory. With nonidentical diagonal partitions, i.e., $\bar{Z}_{11} \neq \bar{Z}_{22}$, MNMF requires larger memory ( $4 / 3$ that of others) to store the near-field interactions.

\section{Calculation of Interactions by MLFMA}

For the solution of scattering problems involving large dielectric objects, we employ MLFMA to efficiently perform the matrix-vector multiplications (MVMs) required by the iterative solvers in $O\left(N N_{L}\right)$ time using $O\left(N N_{L}\right)$ memory, where $N_{L}=$ $O(\log N)$ is the number of levels [26]. We construct a tree structure of $N_{L}$ levels by placing the scatterer in a cubic box and recursively dividing the computational domain into sub-boxes (clusters). Then, MLFMA calculates the interactions between the radiating (basis) and receiving (testing) elements, which are far from each other, in a group-by-group manner consisting of three stages called aggregation, translation, and disaggregation [26]. In each MVM, these stages are performed on the tree structure in a multilevel scheme.

By factorizing the Green's function and performing a diagonalization [27], the interactions in (13)-(16) can be rewritten as

$$
\begin{gathered}
\left\{\begin{array}{c}
K_{l}^{T}[m, n] \\
K_{l}^{N}[m, n] \\
T_{l}^{T}[m, n] \\
T_{l}^{N}[m, n]
\end{array}\right\}=\left(\frac{i k_{l}}{4 \pi}\right)^{2} \int d^{2} \hat{\boldsymbol{k}}\left\{\begin{array}{l}
\boldsymbol{F}_{m C}^{\mathcal{K}, T}\left(\boldsymbol{k}_{l}\right) \\
\boldsymbol{F}_{m, N}^{\mathcal{K}, N}\left(\boldsymbol{k}_{l}\right) \\
\boldsymbol{F}_{m, T}^{\mathcal{T}, T}\left(\boldsymbol{k}_{l}\right) \\
\boldsymbol{F}_{m C}^{\mathcal{T}, N}\left(\boldsymbol{k}_{l}\right)
\end{array}\right\} \\
\cdot \alpha_{L_{l}}\left(\boldsymbol{k}_{l}, \boldsymbol{R}_{C C^{\prime}}\right) \boldsymbol{S}_{C^{\prime} n}\left(\boldsymbol{k}_{l}\right)
\end{gathered}
$$

when the testing and basis functions are far from each other. In (25), the integral is evaluated on the unit sphere, $\boldsymbol{k}_{l}=\hat{\boldsymbol{k}} k_{l}$, and

$$
\alpha_{L_{l}}\left(\boldsymbol{k}_{l}, \boldsymbol{R}_{C C^{\prime}}\right)=\sum_{t=0}^{L_{l}} i^{t}(2 t+1) h_{t}^{(1)}\left(k_{l} R_{C C^{\prime}}\right) P_{t}\left(\hat{\boldsymbol{R}}_{C C^{\prime}} \cdot \hat{\boldsymbol{k}}\right)
$$

is the translation operator expressed in terms of the spherical Hankel function of the first kind $h_{t}^{(1)}$ and the Legendre polynomial $P_{t}$. The translation operator in (26) is employed to translate the radiation pattern of the $n$th basis function in cluster $C^{\prime}$, i.e., $\boldsymbol{S}_{C^{\prime} n}\left(\boldsymbol{k}_{l}\right)$, into incoming fields for the testing functions in cluster $C$. Then, the incoming fields are received by using the receiving pattern of the $m$ th testing function, i.e., $\boldsymbol{F}_{m C}\left(\boldsymbol{k}_{l}\right)$. The distance between the clusters is represented by the vector

$$
\boldsymbol{R}_{C C^{\prime}}=R_{C C^{\prime}} \hat{\boldsymbol{R}}_{C C^{\prime}}=\boldsymbol{r}_{C}-\boldsymbol{r}_{C^{\prime}}
$$

where $\boldsymbol{r}_{C}$ and $\boldsymbol{r}_{C^{\prime}}$ are reference points of the clusters $C$ and $C^{\prime}$, respectively.

The radiation pattern of a basis function $\boldsymbol{b}_{n}(\boldsymbol{r})$ with respect to a reference point $\boldsymbol{r}_{C^{\prime}}$ can be written as

$$
\begin{aligned}
& \boldsymbol{S}_{C^{\prime} n}\left(\boldsymbol{k}_{l}\right) \\
& \quad=\int_{S_{n}} d \boldsymbol{r}^{\prime} \exp \left[-i \boldsymbol{k}_{l} \cdot\left(\boldsymbol{r}^{\prime}-\boldsymbol{r}_{C^{\prime}}\right)\right](\overline{\boldsymbol{I}}-\hat{\boldsymbol{k}} \hat{\boldsymbol{k}}) \cdot \boldsymbol{b}_{n}\left(\boldsymbol{r}^{\prime}\right)
\end{aligned}
$$

where $\bar{I}$ denotes the $3 \times 3$ unit dyad. In contrast to radiation patterns, receiving patterns depend on the type of the operator and the equation. Using a Galerkin scheme, the receiving pattern of a testing function $\boldsymbol{t}_{m}(\boldsymbol{r})$ with respect to a reference point $\boldsymbol{r}_{C}$ can be derived for different operators $(\mathcal{K}$ and $\mathcal{T})$ and equation types ( $\mathrm{T}$ and $\mathrm{N}$ ) as

$$
\begin{aligned}
\boldsymbol{F}_{m C}^{\mathcal{K}, T}\left(\boldsymbol{k}_{l}\right)= & -\hat{\boldsymbol{k}} \times \int_{S_{m}} d \boldsymbol{r} \exp \left[i \boldsymbol{k}_{l} \cdot\left(\boldsymbol{r}-\boldsymbol{r}_{C}\right)\right] \boldsymbol{t}_{m}(\boldsymbol{r}) \\
= & -\hat{\boldsymbol{k}} \times\left\{\boldsymbol{S}_{C m}\left(\boldsymbol{k}_{l}\right)\right\}^{*} \\
\boldsymbol{F}_{m C}^{\mathcal{T}, T}\left(\boldsymbol{k}_{l}\right)= & \int_{S_{m}} d \boldsymbol{r} \exp \left[i \boldsymbol{k}_{l} \cdot\left(\boldsymbol{r}-\boldsymbol{r}_{C}\right)\right](\overline{\boldsymbol{I}}-\hat{\boldsymbol{k}} \hat{\boldsymbol{k}}) \cdot \boldsymbol{t}_{m}(\boldsymbol{r}) \\
= & \left\{\boldsymbol{S}_{C m}\left(\boldsymbol{k}_{l}\right)\right\}^{*} \\
\boldsymbol{F}_{m C}^{\mathcal{T}, N}\left(\boldsymbol{k}_{l}\right)= & \int_{S_{m}} d \boldsymbol{r} \exp \left[i \boldsymbol{k}_{l} \cdot\left(\boldsymbol{r}-\boldsymbol{r}_{C}\right)\right](\overline{\boldsymbol{I}}-\hat{\boldsymbol{k}} \hat{\boldsymbol{k}}) \cdot \boldsymbol{t}_{m}(\boldsymbol{r}) \times \hat{\boldsymbol{n}} \\
\boldsymbol{F}_{m C}^{\mathcal{K}, N}\left(\boldsymbol{k}_{l}\right)= & -\hat{\boldsymbol{k}} \times \int_{S_{m}} d \boldsymbol{r} \exp \left[i \boldsymbol{k}_{l} \cdot\left(\boldsymbol{r}-\boldsymbol{r}_{C}\right)\right] \times \boldsymbol{t}_{m}(\boldsymbol{r}) \times \hat{\boldsymbol{n}} \\
& =\hat{\boldsymbol{k}} \times\left\{\boldsymbol{F}_{m C}^{\mathcal{T}, N}\left(\boldsymbol{k}_{l}\right)\right\}^{*}
\end{aligned}
$$

where "*” represents the complex-conjugate operation. Using the RWG functions, the integrals in (28)-(32) can be calculated analytically.

Similar to the near-field interactions, the radiation and receiving patterns of the basis and testing functions are calculated and stored in memory before the iterations. Since the patterns have only $\theta$ and $\phi$ components, they are stored in spherical coordinates. Using CTF, only one set of patterns is required for each medium $(l=1,2)$, because both of the receiving patterns, $\boldsymbol{F}_{m C}^{\mathcal{K}, T}\left(\boldsymbol{k}_{l}\right)$ and $\boldsymbol{F}_{m C}^{\mathcal{T}, T}\left(\boldsymbol{k}_{l}\right)$, can be obtained from the related radiation pattern $\boldsymbol{S}_{C m}\left(\boldsymbol{k}_{l}\right)$, as indicated in (29) and (30). In other 
words, receiving operations during the MVMs can be performed by using the radiation patterns (instead of the receiving patterns) with small modifications involving a complex conjugation and a cross product with the angular direction $\hat{\boldsymbol{k}}$. However, CNF and MNMF require two sets of patterns, as the receiving patterns $\boldsymbol{F}_{m C}^{\mathcal{K}, N}\left(\boldsymbol{k}_{l}\right)$ and $\boldsymbol{F}_{m C}^{\mathcal{T}, N}\left(\boldsymbol{k}_{l}\right)$ can be derived from each other, but they cannot be obtained directly from the related radiation pattern $\boldsymbol{S}_{C m}\left(\boldsymbol{k}_{l}\right)$ in spherical coordinates. Finally, JMCFIE also requires two sets of patterns, considering all relations in (29)-(32).

In MLFMA, the interactions in (25) are calculated in a multilevel scheme. During the aggregation process, radiation patterns of the clusters are calculated from the bottom to the top of the tree structure. We sample the fields uniformly in the $\phi$ direction and use Gauss-Legendre points in the $\theta$ direction. Then, a total of $\left(L_{l}+1\right) \times\left(2 L_{l}+2\right)$ samples are required for each cluster, where $L_{l}$ is the truncation number for the series in (26). To determine the value of $L_{l}$ for each level, we use the excess bandwidth formula for a one-box-buffer scheme, i.e.,

$$
L_{l} \approx 1.73 k_{l} a+2.16\left(d_{0}\right)^{2 / 3}\left(k_{l} a\right)^{1 / 3}
$$

where $a$ is the box size, and $d_{0}$ is the number of accurate digits [28]. Due to the oscillatory nature of the Helmholtz equation, the truncation number and the sampling rate for the radiation and receiving patterns depend on the size of the clusters with respect to the wavelength associated with the medium. We employ local Lagrange interpolation methods to match the different sampling rates of consecutive levels [29]. After the aggregation stage, translations are performed to obtain the incoming fields for all clusters. Using cubic clusters, there are $O(1)$ different translations in each level, due to the symmetry [30]. Although using the symmetry reduces the number of translation operators significantly, we also need interpolation methods to calculate these operators in $O(N)$ time during the setup stage [31]. After the translations, the disaggregation stage is performed from the top of the tree structure to the lowest level using anterpolations [32]. Finally, the angular integrations in (25) are computed to complete the matrix-vector multiplications.

\section{Block-Diagonal PRECONDITIONING}

To reduce the number of iterations required for the solutions, we apply two types of efficient preconditioners, namely, a twopartition block-diagonal preconditioner (2PBDP) and a fourpartition block-diagonal preconditioner (4PBDP). Matrix equations in (24) can be preconditioned as

$$
\overline{\boldsymbol{P}}^{-1} \cdot\left[\begin{array}{ll}
\overline{\boldsymbol{Z}}_{11} & \overline{\boldsymbol{Z}}_{12} \\
\overline{\boldsymbol{Z}}_{21} & \overline{\boldsymbol{Z}}_{22}
\end{array}\right] \cdot\left[\begin{array}{c}
\boldsymbol{a}_{J} \\
\boldsymbol{a}_{M}
\end{array}\right]=\overline{\boldsymbol{P}}^{-1} \cdot\left[\begin{array}{c}
\boldsymbol{v}_{1}^{\text {inc }} \\
\boldsymbol{v}_{2}^{\text {inc }}
\end{array}\right]
$$

where $\overline{\boldsymbol{P}}$ is a preconditioner matrix. In both 2PBDP and 4PBDP, we use the self interactions of the lowest-level clusters of MLFMA, which are calculated directly during the setup stage of the algorithm. In 2PBDP, we extract the self interactions only from the diagonal partitions of the impedance matrix $\left(\overline{\boldsymbol{Z}}_{11}\right.$ and $\left.\overline{\boldsymbol{Z}}_{22}\right)$ as

$$
\overline{\boldsymbol{P}}_{2 \mathrm{P}}=\left[\begin{array}{cc}
\overline{\boldsymbol{P}}_{11} & 0 \\
0 & \overline{\boldsymbol{P}}_{22}
\end{array}\right]
$$

where $\overline{\boldsymbol{P}}_{11}$ and $\overline{\boldsymbol{P}}_{22}$ are block-diagonal matrices. For 4PBDP, however, we also consider the self interactions in the non-diagonal partitions $\left(\overline{\boldsymbol{Z}}_{12}\right.$ and $\left.\overline{\boldsymbol{Z}}_{21}\right)$ so that

$$
\overline{\boldsymbol{P}}_{4 \mathrm{P}}=\left[\begin{array}{ll}
\overline{\boldsymbol{P}}_{11} & \overline{\boldsymbol{P}}_{12} \\
\overline{\boldsymbol{P}}_{21} & \overline{\boldsymbol{P}}_{22}
\end{array}\right] \text {. }
$$

Since $\overline{\boldsymbol{P}}_{12}$ and $\overline{\boldsymbol{P}}_{21}$ are also block-diagonal matrices, the inverse of $\overline{\boldsymbol{P}}_{4 \mathrm{P}}$ can be evaluated efficiently as [33]

$$
\overline{\boldsymbol{P}}_{4 \mathrm{P}}^{-1}=\left[\begin{array}{ll}
\overline{\boldsymbol{B}}_{11} & \overline{\boldsymbol{B}}_{12} \\
\overline{\boldsymbol{B}}_{21} & \overline{\boldsymbol{B}}_{22}
\end{array}\right]
$$

where

$$
\begin{aligned}
\overline{\boldsymbol{B}}_{11} & =\overline{\boldsymbol{P}}_{11}^{-1} \cdot\left[\overline{\boldsymbol{I}}+\overline{\boldsymbol{P}}_{12} \cdot \overline{\boldsymbol{S}}^{-1} \cdot \overline{\boldsymbol{P}}_{21} \cdot \overline{\boldsymbol{P}}_{11}^{-1}\right] \\
\overline{\boldsymbol{B}}_{12} & =-\overline{\boldsymbol{P}}_{11}^{-1} \cdot \overline{\boldsymbol{P}}_{12} \cdot \overline{\boldsymbol{S}}^{-1} \\
\overline{\boldsymbol{B}}_{21} & =-\overline{\boldsymbol{S}}^{-1} \cdot \overline{\boldsymbol{P}}_{21} \cdot \overline{\boldsymbol{P}}_{11}^{-1} \\
\overline{\boldsymbol{B}}_{22} & =\overline{\boldsymbol{S}}^{-1}
\end{aligned}
$$

and $\overline{\boldsymbol{S}}=\overline{\boldsymbol{P}}_{22}-\overline{\boldsymbol{P}}_{21} \cdot \overline{\boldsymbol{P}}_{11}^{-1} \cdot \overline{\boldsymbol{P}}_{12}$ is the Schur complement of $\overline{\boldsymbol{P}}_{11}$. As presented in Section VI, 2PBDP and 4PBDP reduce the iteration counts significantly, especially for JMCFIE.

\section{RESULTS}

In this section, we investigate the efficiency and accuracy of the solutions when the scattering problems are formulated by CTF, CNF, MNMF, and JMCFIE. For all solutions, near-field interactions are calculated with at most $1 \%$ error, and far-field interactions are computed by MLFMA with three digits of accuracy. Tree structures are constructed by fixing the size of the lowest-level clusters to $0.25 \lambda_{1}$ and using a bottom-up strategy.

\section{A. Efficiency of the MLFMA Solutions}

First, we compare the memory required for the MLFMA solutions of various formulations. We consider a dielectric sphere with a radius of 0.3 meters located in free space and illuminated by a plane wave propagating in the $z$ direction with the electric field polarized in the $x$ direction. The radius of the sphere changes from $0.75 \lambda_{1}$ to $7.5 \lambda_{1}$, where $\lambda_{1}$ is the wavelength in free space. Discretizations with $\lambda_{1} / 10$ triangulations lead to 4142 and 412,998 unknowns, respectively, for radii $0.75 \lambda_{1}$ and $7.5 \lambda_{1}$. The relative permittivity of the sphere is $\epsilon_{r}=2.0$. Fig. 1 presents the peak memory required for the MLFMA solutions with respect to the number of unknowns. The peak memory depends on the formulation type mainly because of the different storage requirements for the near-field interactions (identical or nonidentical diagonal partitions) and the far-field patterns of the basis and testing functions (one set or two sets of patterns). Having identical diagonal partitions and using one set of far-field patterns, CTF requires less memory than the other formulations. CNF and JMCFIE require two sets of far-field patterns, and their memory usage is larger than CTF. Finally, MNMF has nonidentical diagonal partitions and requires two sets of far-field patterns, leading to a larger memory usage than CTF, CNF, and JMCFIE. Although the memory is not critical for small problems, it becomes more important as the problem size 
TABLE I

Number of CGS Iterations to Reduce the Residual ERror Below $10^{-3}$ For the Solution of Sphere Problems With $\epsilon_{r}=4.0$

\begin{tabular}{|c|c|c|c|c|c|c|c|c|c|c|c|c|c|}
\hline \multicolumn{2}{|c|}{ Sphere Problems } & \multicolumn{3}{|c|}{$\mathrm{CTF}$} & \multicolumn{3}{|c|}{$\mathrm{CNF}$} & \multicolumn{3}{|c|}{ MNMF } & \multicolumn{3}{|c|}{ JMCFIE } \\
\hline Radius & Unknowns & NP & 2PBDP & 4PBDP & NP & 2PBDP & 4PBDP & NP & 2PBDP & 4PBDP & NP & 2PBDP & 4PBDP \\
\hline $0.75 \lambda_{1}$ & 4152 & 102 & 344 & 325 & 48 & 47 & 32 & 34 & 26 & 19 & 46 & 34 & 28 \\
\hline $1.00 \lambda_{1}$ & 7446 & 245 & 623 & 557 & 87 & 52 & 42 & 46 & 30 & 30 & 94 & 48 & 37 \\
\hline $1.50 \lambda_{1}$ & 16,728 & 259 & 1145 & 959 & 184 & 115 & 76 & 66 & 51 & 32 & 129 & 77 & 63 \\
\hline $2.00 \lambda_{1}$ & 29,742 & 213 & 1317 & 1183 & 272 & 182 & 90 & 66 & 54 & 46 & 122 & 86 & 54 \\
\hline $3.00 \lambda_{1}$ & 65,724 & 678 & $\mathrm{NC}$ & $\mathrm{NC}$ & 305 & 278 & 128 & 144 & 111 & 108 & 173 & 189 & 113 \\
\hline $6.00 \lambda_{1}$ & 264,006 & 417 & $\mathrm{NC}$ & $\mathrm{NC}$ & $\mathrm{NC}$ & 1583 & 755 & 332 & 234 & 174 & 414 & 276 & 148 \\
\hline $7.50 \lambda_{1}$ & 412,998 & 369 & $\mathrm{NC}$ & $\mathrm{NC}$ & 1153 & 1005 & 468 & 380 & 303 & 321 & 269 & 230 & 123 \\
\hline
\end{tabular}

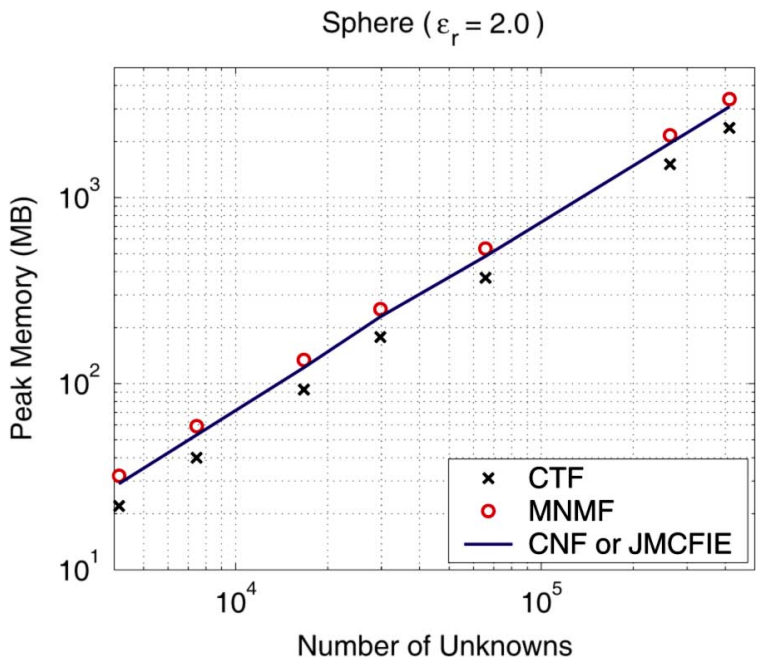

Fig. 1. Peak memory required for the MLFMA solutions of scattering problems involving a sphere with a relative permittivity of 2.0. The radius of the sphere is in the range from $0.75 \lambda_{1}$ to $7.5 \lambda_{1}$, where $\lambda_{1}$ is the wavelength in free space.

grows. For example, when the radius is $7.5 \lambda_{1}$, the peak memory is $2370 \mathrm{MB}$ and $3385 \mathrm{MB}$ for CTF and MNMF, respectively.

Next, we focus on the processing time required for the MLFMA solutions of dielectric problems. Once again, we consider the solution of scattering problems involving a dielectric sphere with a radius of 0.3 meters located in free space and illuminated by a plane wave propagating in the $z$ direction with the electric field polarized in the $x$ direction. As the problem size gets larger, the setup time becomes negligible, compared with the time required for the iterations. Then, the processing time of the MLFMA solutions is directly proportional to the iteration counts. Table I lists the number of conjugate-gradient-squared (CGS) iterations to reduce the residual error below $10^{-3}$ when the radius of the sphere is in the range from $0.75 \lambda_{1}$ to $7.5 \lambda_{1}$ and $\epsilon_{r}=4.0$. For all formulations, we apply two efficient preconditioners, i.e., $2 \mathrm{PBDP}$ and $4 \mathrm{PBDP}$, in addition to the no-preconditioner (NP) case. Our comments for the iteration counts in Table I are as follows.

- Preconditioning the matrix equations with 2PBDP and 4PBDP does not accelerate the iterative convergence for CTF. In fact, these low-cost preconditioners decelerate the convergence and increase the number of iterations for this formulation. Especially for large problems, convergence cannot be achieved within 2000 iterations, when 2PBDP and 4PBDP are used for CTF. These are denoted as "no convergence (NC)" in Table I. A negative effect of the efficient preconditioners was also observed for other $\mathrm{T}$ formulations, such as T-EFIE for the solution of PEC objects [34]. However, 2PBDP and 4PBDP significantly accelerate the iterative convergence for the $\mathrm{N}$ formulations and JMCFIE.

- Although they are both $\mathrm{N}$ formulations, iteration counts for CNF and MNMF differ significantly; convergence is consistently faster for MNMF. For large problems, CNF fails to provide quick convergence even when compared to CTF without preconditioning.

- Using 4PBDP provides faster convergence than 2PBDP, especially for JMCFIE. This is due to the strong non-diagonal partitions of JMCFIE, in addition to its strong diagonal partitions involving well-tested identity operators.

- For small problems, iteration counts for MNMF are lower than JMCFIE. This can be observed in Table I, when the radius of the sphere is in the range from $0.75 \lambda_{1}$ to $3 \lambda_{1}$. For larger problems, however, the convergence for JMCFIE becomes faster than the convergence for MNMF. When the radius of the sphere is $7.5 \lambda_{1}$, iteration counts are consistently lower for JMCFIE with and without preconditioning.

To further compare the dielectric formulations in terms of efficiency, we consider scattering problems involving a dielectric cube with edges of $4 \lambda_{1}$ located in free space. Similar to sphere problems, the cube is illuminated by a plane wave propagating in the $z$ direction with the electric field polarized in the $x$ direction. Discretization of the problem with $\lambda_{1} / 10$ triangulation leads to 64,548 unknowns. The relative permittivity of the cube changes from 2.0 to 16.0. Fig. 2(a) depicts the number of CGS iterations with respect to the contrast of the cube, i.e., $\left(\epsilon_{r}-1\right)$, to reduce the residual error below $10^{-3}$. For small contrasts, CTF has the slowest convergence, while the $\mathrm{N}$ formulations, i.e., $\mathrm{CNF}$ and MNMF, offer the fastest convergence. As the contrast increases, however, convergence of CNF decelerates significantly and this formulation has the poorest convergence when $\epsilon_{r}=16.0$. Fig. 2(a) also shows that MNMF provides the most efficient solutions and the number of iterations for this formulation is almost constant when the contrast increases from 3.0 to 15.0. Fig. 2(b) presents the iteration counts when 4PBDP 


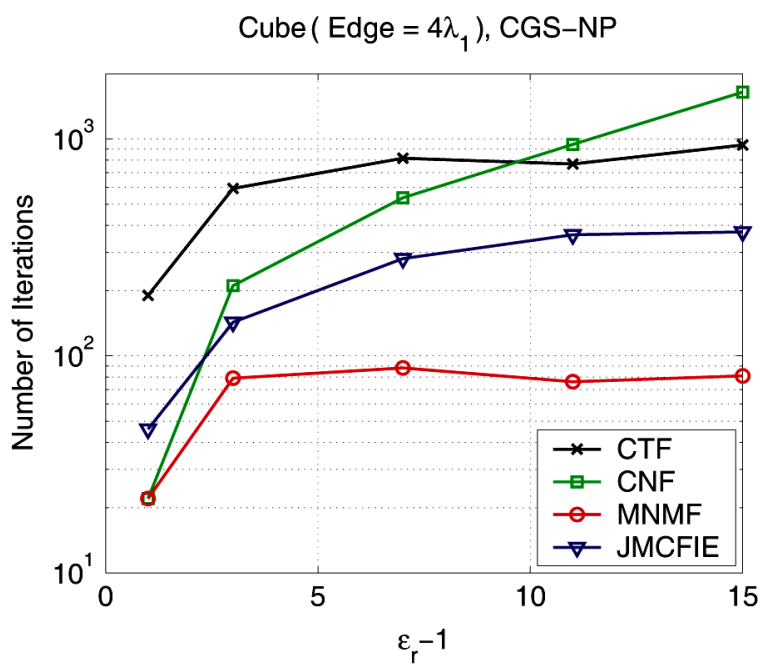

(a)

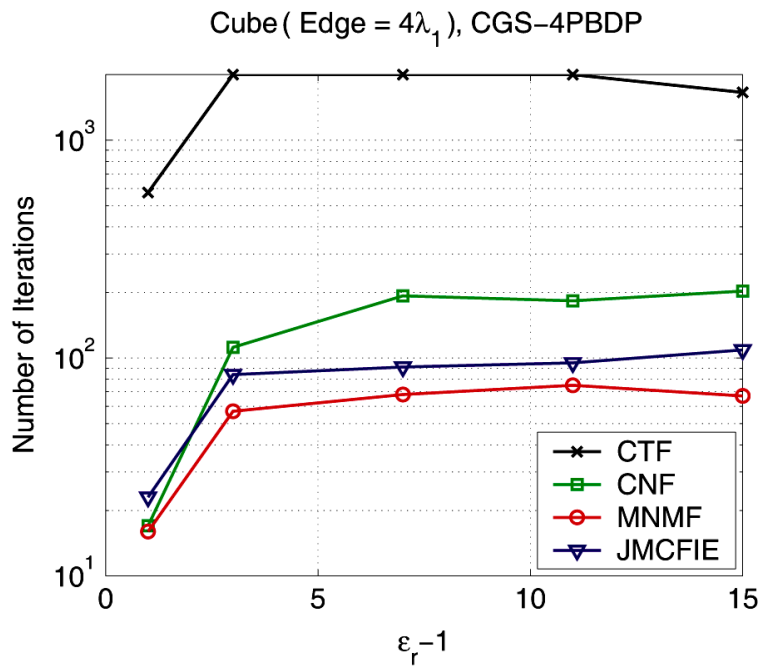

(b)

Fig. 2. Iteration counts for the solution of scattering problems involving a cube with edges of $4 \lambda_{1}$, where $\lambda_{1}$ is the wavelength in free space. The relative permittivity $\left(\epsilon_{r}\right)$ of the cube changes from 2.0 to 16.0 . Iterative solutions are performed by CGS (a) without preconditioning and (b) accelerated with 4 PBDP.

is used to accelerate the convergence of the solutions. Similar to sphere problems, CTF solutions are decelerated with 4PBDP and the convergence cannot be achieved within 2000 iterations for contrasts from 3.0 to 11.0. Comparing Fig. 2(b) with (a), we also observe that 4PBDP significantly reduces the number of iterations for CNF and JMCFIE, but it provides less improvement for MNMF. Using 4PBDP, MNMF still has the lowest iteration counts, but the efficiency of JMCFIE becomes close to the efficiency of MNMF.

Table I and Fig. 2 show that MNMF and JMCFIE are the most appropriate formulations for the efficient solutions of large dielectric problems. These formulations are further investigated by considering both various preconditioning schemes and different iterative algorithms to improve the efficiency of the solutions. As an example, Fig. 3 presents the iteration counts for the sphere problems when $\epsilon_{r}=2.0$. In addition to CGS with no preconditioning (NP) and with 4PBDP, we also

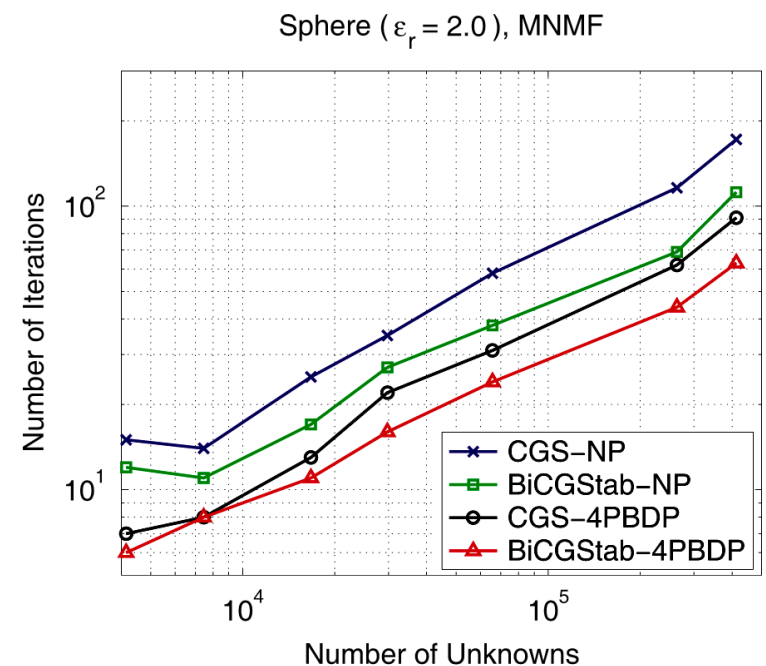

(a)

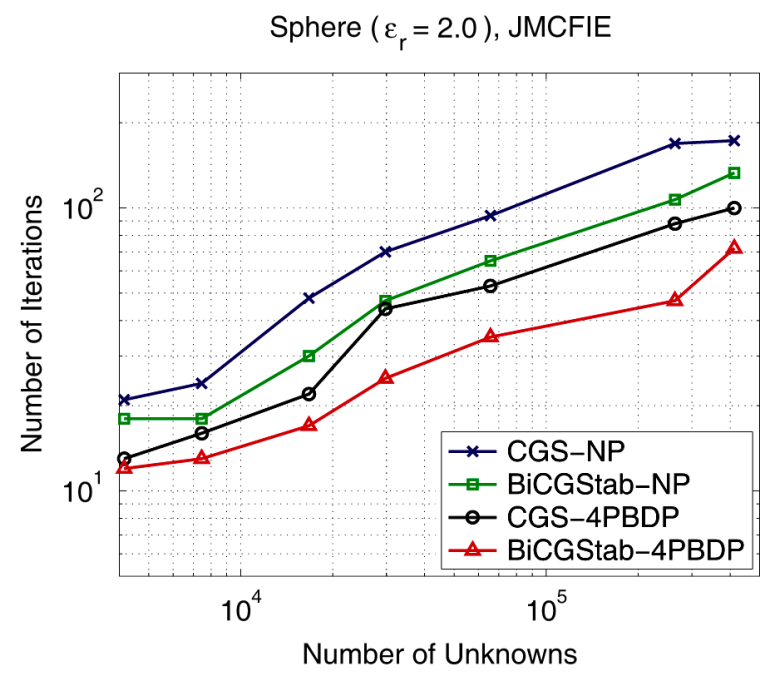

(b)

Fig. 3. Iteration counts for the solution of scattering problems involving a sphere with a relative permittivity of 2.0, when the problems are formulated with (a) MNMF and (b) JMCFIE. The radius of the sphere is in the range from $0.75 \lambda_{1}$ to $7.5 \lambda_{1}$, where $\lambda_{1}$ is the wavelength in free space.

present the solutions obtained by employing a biconjugate-gradient-stabilized (BiCGStab) algorithm. BiCGStab is known to provide rapid convergence for $\mathrm{N}$ and combined formulations [35]. Fig. 3 shows that the number of iterations is reduced for both MNMF and JMCFIE, if BiCGStab is employed, instead of CGS.

Finally, MNMF and JMCFIE are compared for the solution of very large dielectric problems. Fig. 4(a) presents the iteration counts for the sphere problems $\left(\epsilon_{r}=2.0\right)$, when the solutions are performed by BiCGStab accelerated with 4PBDP. This time, the frequency is extended to $20 \mathrm{GHz}$ and the radius of the sphere grows up to $20 \lambda_{1}$. At $20 \mathrm{GHz}$, discretization of the sphere with $\lambda_{1} / 10$ triangulation leads to 2,925,708 unknowns. Fig. 4(a) shows that solutions with JMCFIE become significantly faster than MNMF for large problems. Using JMCFIE, we are able to solve a 3-million-unknown problem, which is one of the largest integral-equation problems involving dielectric objects ever solved. Fig. 4(b) presents the results of a 


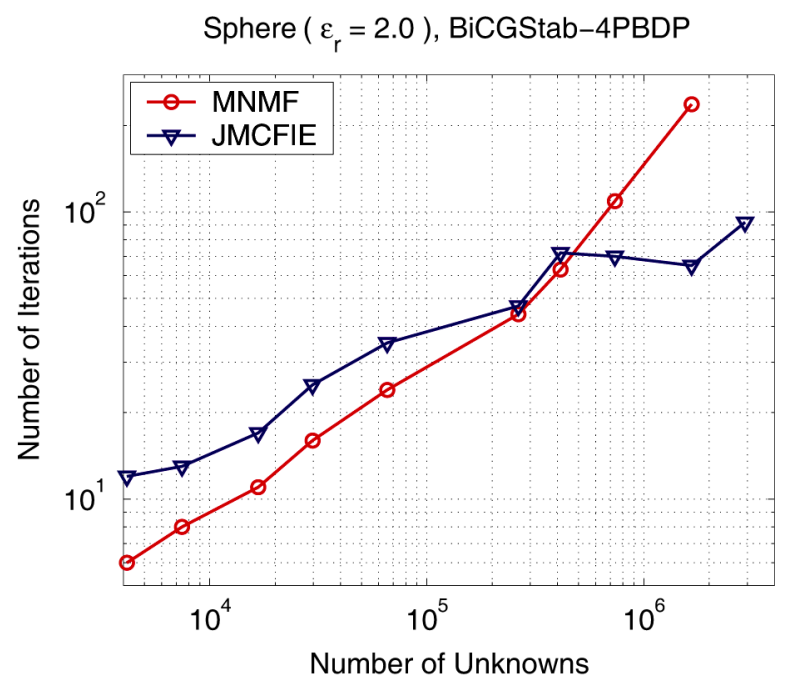

(a)

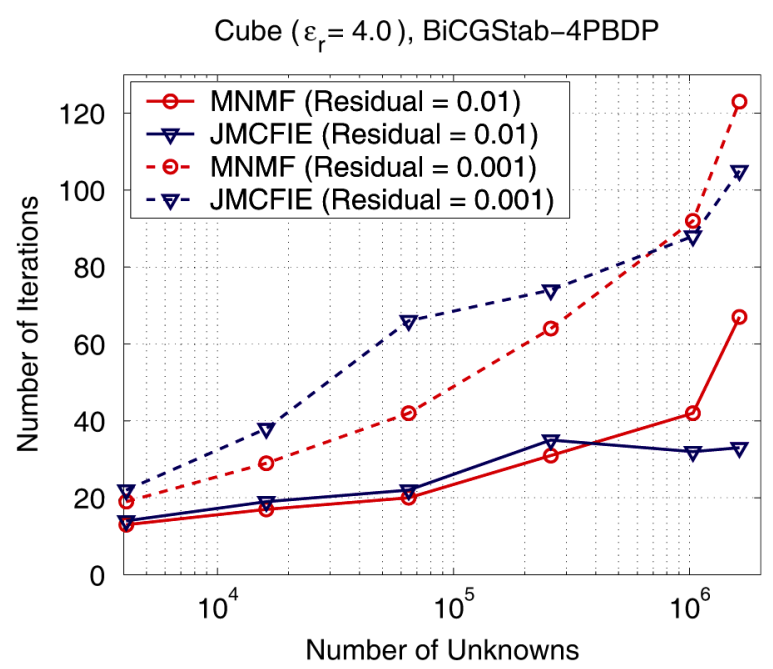

(b)

Fig. 4. Iteration counts for the solution of scattering problems involving (a) a sphere with a relative permittivity of 2.0 and (b) a cube with a relative permittivity of 4.0. The radius of the sphere is in the range from $0.75 \lambda_{1}$ to $20 \lambda_{1}$ and the edge length of the cube is in the range from $\lambda_{1}$ to $20 \lambda_{1}$, where $\lambda_{1}$ is the wavelength in free space. Iterative solutions are performed by employing BiCGStab accelerated with 4 PBDP.

similar experiment, where scattering problems involving a dielectric cube $\left(\epsilon_{r}=4.0\right)$ is solved by using BiCGStab accelerated with $4 \mathrm{PBDP}$. The size of the cube changes from $\lambda_{1}$ to $20 \lambda_{1}$, where $\lambda_{1}$ is the wavelength in free space. The number of unknowns due to $\lambda_{1} / 10$ triangulations is in the range from 4104 to $1,624,320$. Iteration counts required for both $10^{-2}$ and $10^{-3}$ residual errors are plotted with respect to the number of unknowns. Similar to the previous case, solutions with JMCFIE become faster than MNMF for large problems.

In general, JMCFIE leads to more efficient solutions than MNMF, when the problem size is sufficiently large. Our investigations further show that the better performance of JMCFIE becomes more evident when a problem involves complicated targets. As an example, Fig. 5 presents the results of a problem involving a 5-layer periodic structure in free space excited by a Hertzian dipole. Dimensions of the structure and the position of the source are detailed in Fig. 5(a). Discretization of

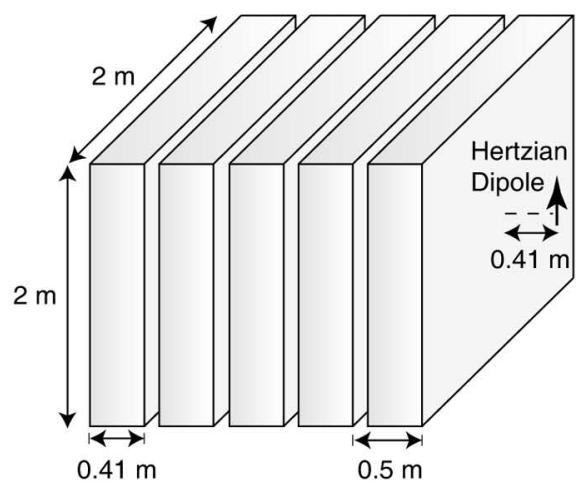

(a)

Periodic Slabs, BiCGStab-BP

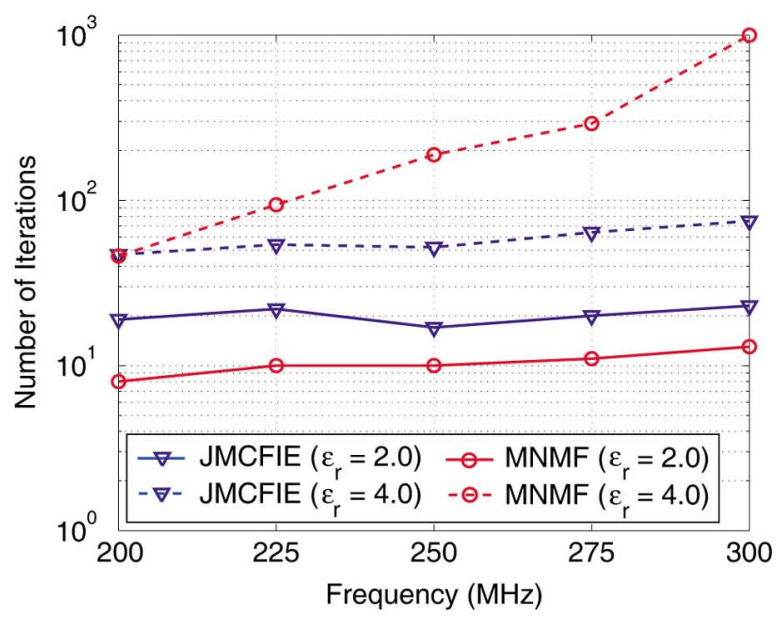

(b)

Fig. 5. (a) A 5-layer periodic dielectric structure illuminated by a Hertzian dipole. (b) Iteration counts (using BiCGStab accelerated with 4PBDP) for the solution of the problem in Fig. 5(a) when the frequency changes from $200 \mathrm{MHz}$ to $300 \mathrm{MHz}$, and the relative permittivity $\left(\epsilon_{r}\right)$ of the structure is 2.0 and 4.0 .

the structure with $10 \mathrm{~cm}$ triangulation size leads to 38,700 unknowns. We consider two different values for the relative permittivity of the structure, i.e., $\epsilon_{r}=2.0$ and $\epsilon_{r}=4.0$. Fig. 5(b) depicts the iteration counts for $10^{-3}$ residual error as a function of frequency from $200 \mathrm{MHz}$ to $300 \mathrm{MHz}$, when the problems are solved by using BiCGStab accelerated with 4PBDP. We observe that MNMF offers faster solutions when $\epsilon_{r}=2.0$, and the number of iterations is halved compared to JMCFIE. When $\epsilon_{r}=4.0$, however, the number of iterations for MNMF increases rapidly as the frequency changes from $200 \mathrm{MHz}$ to $300 \mathrm{MHz}$. At $300 \mathrm{MHz}$, convergence cannot be achieved within 1000 iterations by using MNMF. On the other hand, JMCFIE is more stable in the same frequency range, and it provides significantly faster solutions when $\epsilon_{r}=4.0$ and the frequency is larger than $200 \mathrm{MHz}$.

\section{B. Accuracy of the MLFMA Solutions}

Fig. 6(a) depicts the bistatic radar cross section (RCS) values for a sphere with a radius of $3 \lambda_{1}$ and $\epsilon_{r}=2.0$. Normalized RCS $\left(\mathrm{RCS} / \lambda_{1}^{2}\right.$ in $\left.\mathrm{dB}\right)$ is plotted as a function of the observation angle from $0^{\circ}$ to $180^{\circ}$ on the $z-x$ plane, where $0^{\circ}$ corresponds to the forward-scattering direction. Fig. 6(a) shows that the computational values calculated by using CTF are in agreement with the 


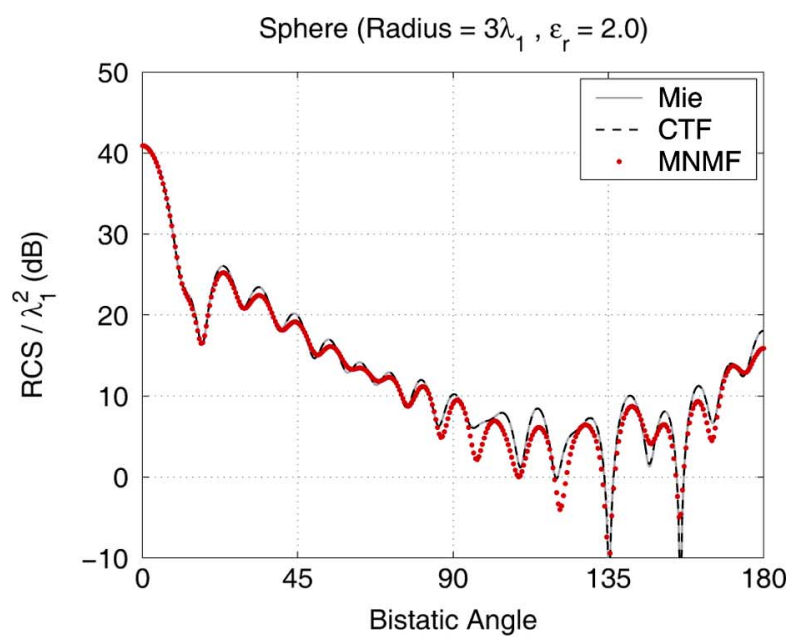

(a)

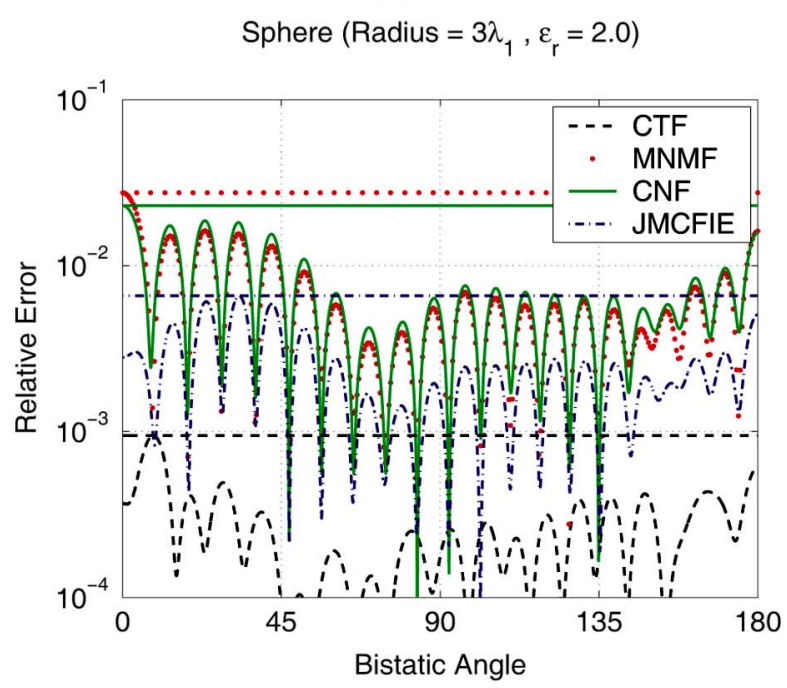

(b)

Fig. 6. (a) Normalized bistatic RCS (RCS/ $\left.\lambda_{1}^{2}\right)$ of a sphere with a radius of $3 \lambda_{1}$ and with a relative permittivity of 2.0. (b) Relative error defined in (42) for different formulations as a function of the bistatic angle.

analytical curve obtained by a Mie-series solution. Although the results obtained by MNMF are also close to the analytical curve, they are significantly inaccurate compared to CTF. For more quantitative information, Fig. 6(b) presents the relative error in the computational results with respect to the reference analytical solution. In addition to CTF and MNMF, we also consider the error for CNF and JMCFIE. The relative error as a function of bistatic angle $\theta$ is defined as

$$
e(\theta)=\frac{\left|f_{C}(\theta)-f_{A}(\theta)\right|}{\max _{\theta}\left|f_{A}(\theta)\right|}
$$

where $f_{C}$ and $f_{A}$ are the computational and analytical values of the far-zone co-polar electric field, i.e.,

$$
f(\theta)=\lim _{r \rightarrow \infty}\left\{r E_{\theta}(r, \theta, \phi=0)\right\} .
$$

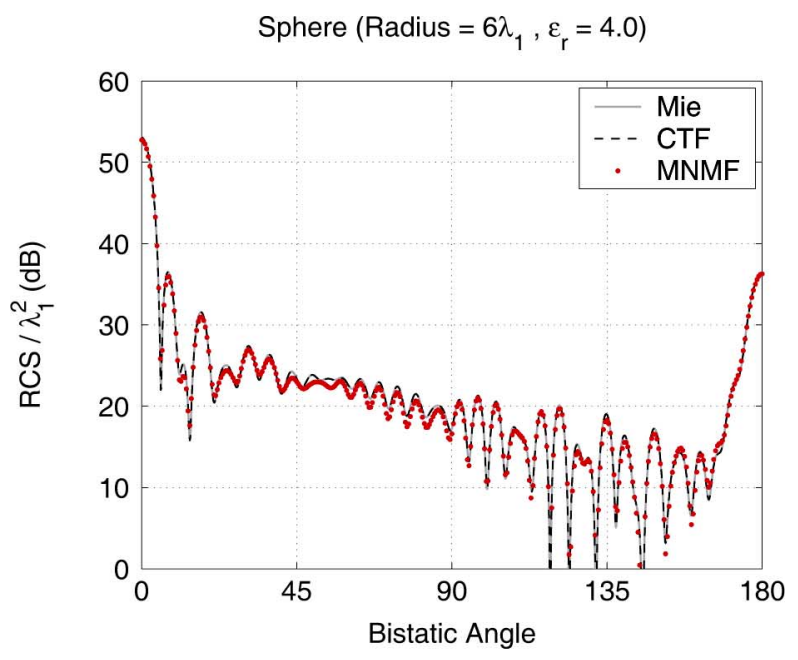

(a)

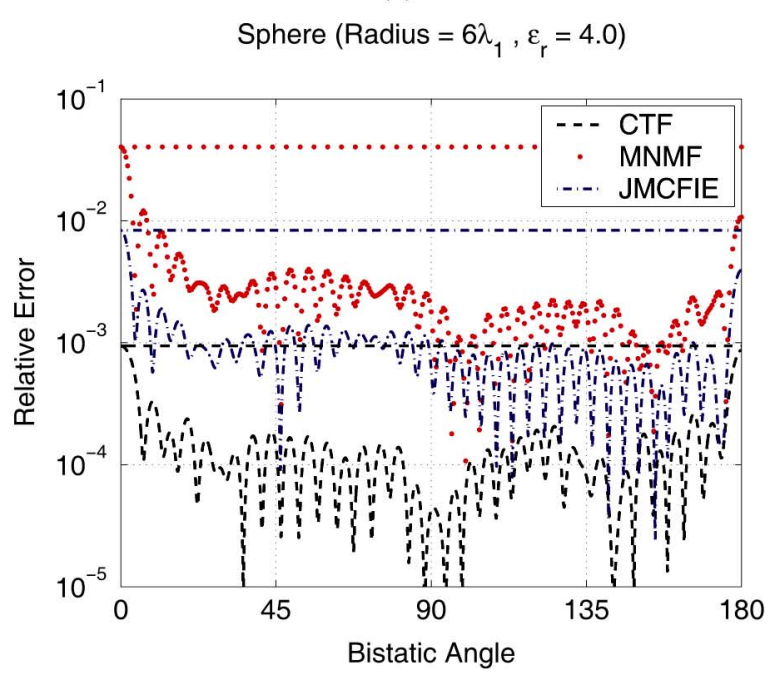

(b)

Fig. 7. (a) Normalized bistatic RCS $\left(\mathrm{RCS} / \lambda_{1}^{2}\right)$ of a sphere with a radius of $6 \lambda_{1}$ and with a relative permittivity of 4.0. (b) Relative error defined in (42) for different formulations as a function of the bistatic angle. CNF is omitted in this figure since its accuracy is very close to that of MNMF, as depicted in Fig. 6(b).

The maximum value of the relative error is also indicated by a horizontal line in the figure for each formulation. Fig. 6(b) shows that CTF provides the most accurate results, while the $\mathrm{N}$ formulations (MNMF and CNF) are significantly inaccurate compared to CTF. JMCIE is also worse than CTF, but it is more accurate than the $\mathrm{N}$ formulations. In Fig. 7, we present the bistatic RCS values and the relative error for a sphere with a radius of $6 \lambda_{1}$ and $\epsilon_{r}=4.0$. The results are very similar to the previous case.

In general, the $\mathrm{N}$ formulations MNMF and CNF are consistently inaccurate, compared to CTF and JMCFIE. Similar observations on the accuracy of the $\mathrm{N}$ formulations were made for small and moderate-size PEC [36]-[39] and dielectric objects [14], as well as large PEC objects [40]. It was also shown that the accuracy of the $\mathrm{N}$ formulations for PEC objects, e.g., N-MFIE, could be improved by employing more appropriate basis functions, instead of the RWG functions [39]-[44]. Improving the accuracy of the $\mathrm{N}$ formulations by employing the 


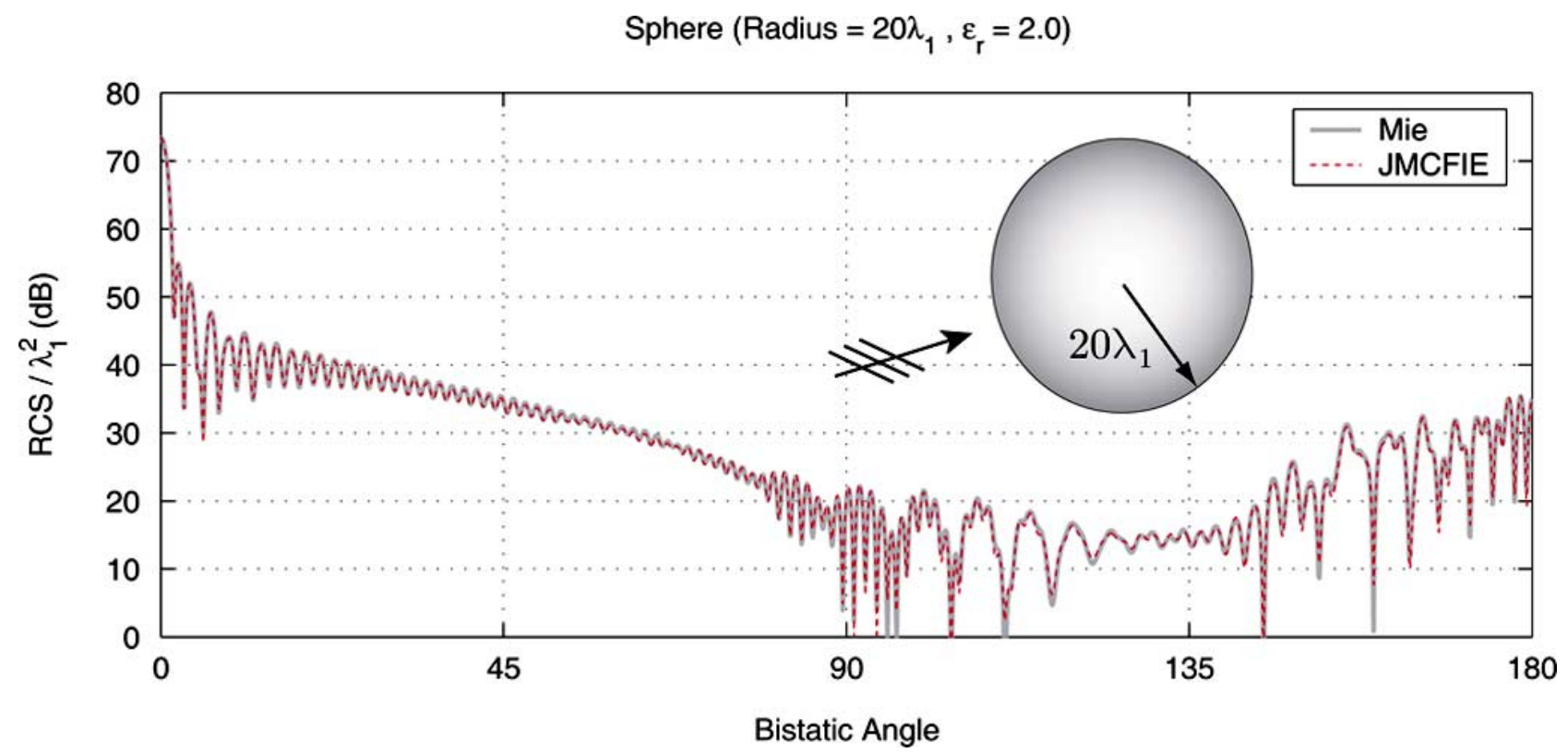

Fig. 8. Normalized bistatic RCS (RCS/ $\lambda_{1}^{2}$ ) of a sphere with a radius of $20 \lambda_{1}$ and with a relative permittivity of 2.0.

linear-linear (LL) basis functions [45] is presented for both perfectly conducting and dielectric objects [14], [46], [47]. In general, the error in the $\mathrm{N}$ formulations is caused by the well-tested identity terms discretized with low-order functions, such as the RWG functions [48]. As demonstrated in Figs. 6 and 7, this error can be significant even for large dielectric objects with smooth surfaces.

Being a combination of $\mathrm{CTF}$ and CNF, solutions with JMCFIE are contaminated with the inaccuracy of CNF. Therefore, CTF is preferable to JMCFIE in terms of accuracy. However, JMCFIE is more suitable for the solution of large problems, which cannot easily be obtained with CTF. For example, Fig. 8 presents the bistatic RCS values for a sphere with a radius of $20 \lambda_{1}$ and $\epsilon_{r}=2.0$ discretized with 2,925,708 unknowns. The computational values obtained with JMCFIE are close to the analytical Mie-series solution, and the maximum relative error is $2.4 \%$. This problem cannot be solved in a reasonable number of iterations when it is formulated with CTF, CNF, or MNMF.

\section{CONCLUDING REMARKS}

In this paper, we investigate surface-integral equations for the fast and accurate solutions of large scattering problems with MLFMA. We specifically consider CTF, CNF, MNMF, and JMCFIE, which were recently developed for stable solutions of dielectric problems. Our observations can be summarized as follows.

- Although MNMF provides the fastest iterative convergence for small problems, JMCFIE has the smallest iteration counts when the problem size is large. This result is similar to the better convergence provided by T-N-CFIE over N-MFIE for perfectly conducting objects.
- In terms of iteration counts, JMCFIE is more stable than MNMF, especially when the solutions are accelerated with 4PBDP. MNMF may fail to provide efficient solutions, when the problems involve complicated targets.

- Iteration counts for CNF grow rapidly as the problem size increases. For large problems, the efficiency of CNF is even worse than CTF. This may not be predictable, because $\mathrm{CNF}$ is an $\mathrm{N}$ formulation and contains well-tested identity operators.

- Using the RWG functions, MNMF and CNF are inaccurate, compared to CTF. Being a combination of CTF and CNF, JMCFIE is also more inaccurate than CTF. However, its accuracy is significantly better than the accuracy of the $\mathrm{N}$ formulations, i.e., MNMF and CNF. The discrepancy among the results of the $\mathrm{N}$ formulations, JMCFIE, and CTF is visible even for large dielectric objects with smooth surfaces.

Finally, considering the trade-off for accuracy and efficiency, CTF and JMCFIE are preferable, respectively. The accuracy of JMCFIE can be improved by employing higher-order basis functions, such as the LL functions, while the efficiency of CTF can be improved by using robust preconditioners.

\section{REFERENCES}

[1] A. J. Poggio and E. K. Miller, "Integral equation solutions of three-dimensional scattering problems," in Computer Techniques for Electromagnetics, R. Mittra, Ed. Oxford, U.K.: Pergamon Press, 1973, ch. 4.

[2] R. F. Harrington, Field Computation by Moment Methods. New York: Macmillan, 1968.

[3] C. Müller, Foundations of the Mathematical Theory of Electromagnetic Waves. New York: Springer, 1969.

[4] T. K. Wu and L. L. Tsai, "Scattering from arbitrarily-shaped lossy dielectric bodies of revolution," Radio Sci., vol. 12, pp. 709-718, Sep.Oct. 1977. 
[5] Y. Chang and R. F. Harrington, "A surface formulation for characteristic modes of material bodies," IEEE Trans. Antennas Propag., vol. AP-25, pp. 789-795, Nov. 1977.

[6] J. R. Mautz and R. F. Harrington, "Electromagnetic scattering from a homogeneous material body of revolution," AE $\ddot{U}$, vol. 33, pp. 71-80, Feb. 1979.

[7] R. F. Harrington, "Boundary integral formulations for homogeneous material bodies," J. Electromagn. Waves Applicat., vol. 3, no. 1, pp. $1-15,1989$.

[8] S. M. Rao and D. R. Wilton, "E-field, H-field, and combined field solution for arbitrarily shaped three-dimensional dielectric bodies," Electromagn., vol. 10, no. 4, pp. 407-421, Oct.-Dec. 1990.

[9] X.-Q. Sheng, J.-M. Jin, J. Song, W. C. Chew, and C.-C. Lu, "Solution of combined-field integral equation using multilevel fast multipole algorithm for scattering by homogeneous bodies," IEEE Trans. Antennas Propag., vol. 46, no. 11, pp. 1718-1726, Nov. 1998.

[10] P. Ylä-Oijala, "Application of a novel CFIE for electromagnetic scattering by dielectric objects," Microw. Opt. Technol., vol. 35, no. 10, pp. $3-5$, Oct. 2002.

[11] T. W. Lloyd, J. M. Song, G. Kang, and C.-C. Lu, "Numerical study of surface integral formulations for homogeneous bodies," in Proc. IEEE Antennas and Propag. Soc. Int. Symp., 2004, pp. 3895-3898.

[12] P. Ylä-Oijala and M. Taskinen, "Application of combined field integral equation for electromagnetic scattering by dielectric and composite objects," IEEE Trans. Antennas Propag., vol. 53, no. 3, pp. 1168-1173, Mar. 2005.

[13] P. Ylä-Oijala and M. Taskinen, "Well-conditioned Müller formulation for electromagnetic scattering by dielectric objects," IEEE Trans. Antennas Propag., vol. 53, no. 10, pp. 3316-3323, Oct. 2005.

[14] P. Ylä-Oijala, M. Taskinen, and S. Järvenpää, "Surface integral equation formulations for solving electromagnetic scattering problems with iterative methods," Radio Sci., vol. 40, Nov. 2005, RS6002, doi:10. 1029/2004RS003169.

[15] T. W. Lloyd, J. M. Song, and M. Yang, "Numerical study of surface integral formulations for low-contrast objects," IEEE Antennas Wireless Propag. Lett., vol. 4, pp. 482-485, 2005.

[16] P. Ylä-Oijala and M. Taskinen, "Improving conditioning of electromagnetic surface integral equations using normalized field quantities," IEEE Trans. Antennas Propag., vol. 55, no. 1, pp. 178-185, Jan. 2007.

[17] J. Song, C.-C. Lu, and W. C. Chew, "Multilevel fast multipole algorithm for electromagnetic scattering by large complex objects," IEEE Trans. Antennas Propag., vol. 45, no. 10, pp. 1488-1493, Oct. 1997.

[18] P. Ylä-Oijala, M. Taskinen, and J. Sarvas, "Surface integral equation method for general integral equation method for general composite metallic and dielectric structures with junctions," Progress In Electromagn. Res. (PIER), vol. 52, pp. 81-108, 2005, doi:10.2528/PIER04071301.

[19] S. M. Rao, D. R. Wilton, and A. W. Glisson, "Electromagnetic scattering by surfaces of arbitrary shape," IEEE Trans. Antennas Propag., vol. AP-30, no. 3, pp. 409-418, May 1982.

[20] R. E. Hodges and Y. Rahmat-Samii, "The evaluation of MFIE integrals with the use of vector triangle basis functions," Microw. Opt. Technol. Lett., vol. 14, no. 1, pp. 9-14, Jan. 1997.

[21] D. A. Dunavant, "High degree efficient symmetrical Gaussian quadrature rules for the triangle," Int. J. Numer. Meth. Eng., vol. 21, pp. $1129-1148,1985$.

[22] L. Gürel and Ö. Ergül, "Singularity of the magnetic-field integral equation and its extraction," IEEE Antennas Wireless Propag. Lett., vol. 4, pp. 229-232, 2005.

[23] Ö. Ergül, "Fast multipole method for the solution of electromagnetic scattering problems," M.S. thesis, Bilkent Univ., Ankara, Turkey, Jun. 2003.

[24] P. Y. -Oijala and M. Taskinen, "Calculation of CFIE impedance matrix elements with RWG and $\hat{\boldsymbol{n}} \times$ RWG functions," IEEE Trans. Antennas Propag., vol. 51, no. 8, pp. 1837-1846, Aug. 2003.

[25] R. D. Graglia, "On the numerical integration of the linear shape functions times the 3-D green's function or its gradient on a plane triangle," IEEE Trans. Antennas Propag., vol. 41, no. 10, pp. 1448-1455, Oct. 1993.

[26] W. C. Chew, J.-M. Jin, E. Michielssen, and J. Song, Fast and Efficient Algorithms in Computational Electromagnetics. Boston, MA: Artech House, 2001.
[27] R. Coifman, V. Rokhlin, and S. Wandzura, "The fast multipole method for the wave equation: A pedestrian prescription," IEEE Antennas Propag. Mag., vol. 35, no. 3, pp. 7-12, Jun. 1993.

[28] S. Koc, J. M. Song, and W. C. Chew, "Error analysis for the numerical evaluation of the diagonal forms of the scalar spherical addition theorem," SIAM J. Numer. Anal., vol. 36, no. 3, pp. 906-921, 1999.

[29] Ö. Ergül and L. Gürel, "Enhancing the accuracy of the interpolations and anterpolations in MLFMA," IEEE Antennas Wireless Propag. Lett., vol. 5, pp. 467-470, 2006.

[30] S. Velamparambil, W. C. Chew, and J. Song, "10 million unknowns: Is it that big?," IEEE Antennas Propag. Mag., vol. 45, no. 2, pp. 43-58, Apr. 2003.

[31] Ö. Ergül and L. Gürel, "Optimal interpolation of translation operator in multilevel fast multipole algorithm," IEEE Trans. Antennas Propag., vol. 54, no. 12, pp. 3822-3826, Dec. 2006.

[32] A. Brandt, "Multilevel computations of integral transforms and particle interactions with oscillatory kernels," Comp. Phys. Comm., vol. 65, pp. 24-38, Apr. 1991.

[33] L. Gürel and W. C. Chew, "Recursive algorithms for calculating the scattering from N strips or patches," IEEE Trans. Antennas Propag., vol. AP-38, pp. 507-515, Apr. 1990.

[34] L. Gürel and Ö. Ergül, "Comparisons of FMM implementations employing different formulations and iterative solvers," in Proc. IEEE Antennas and Propag. Soc. Int. Symp., 2003, vol. 1, pp. 19-22.

[35] L. Gürel and Ö. Ergül, "Fast and accurate solutions of integral-equation formulations discretised with tens of millions of unknowns," Electron. Lett., vol. 43, no. 9, pp. 499-500, Apr. 2007.

[36] Ö. Ergül and L. Gürel, "Investigation of the inaccuracy of the MFIE discretized with the RWG basis functions," in Proc. IEEE Antennas and Propag. Soc. Int. Symp., 2004, vol. 3, pp. 3393-3396.

[37] Ö. Ergül and L. Gürel, "Improved testing of the magnetic-field integral equation," IEEE Microw. Wireless Comp. Lett., vol. 15, no. 10, pp. 615-617, Oct. 2005.

[38] Ö. Ergül and L. Gürel, "Solid-angle factor in the magnetic-field integral equation,” Microw. Opt. Technol. Lett., vol. 45, no. 5, pp. 452-456, Jun. 2005.

[39] Ö. Ergül and L. Gürel, "Improving the accuracy of the MFIE with the choice of basis functions," in Proc. IEEE Antennas and Propag. Soc. Int. Symp., 2004, vol. 3, pp. 3389-3392.

[40] Ö. Ergül and L. Gürel, "On the accuracy of MFIE and CFIE in the solution of large electromagnetic scattering problems," presented at the Eur. Conf. Antennas and Propag. (EuCAP), 2006.

[41] E. Ubeda and J. M. Rius, "MFIE MOM-formulation with curl-conforming basis functions and accurate kernel integration in the analysis of perfectly conducting sharp-edged objects," Microw. Opt. Technol. Lett., vol. 44, no. 4, pp. 354-358, Feb. 2005.

[42] E. Ubeda and J. M. Rius, "Monopolar divergence-conforming and curl-conforming low-order basis functions for the electromagnetic scattering analysis," Microw. Opt. Technol. Lett., vol. 46, no. 3, pp. 237-241, Aug. 2005.

[43] E. Ubeda and J. M. Rius, "Novel monopolar MFIE MoM-discretization for the scattering analysis of small objects," IEEE Trans. Antennas Propag., vol. 54, no. 1, pp. 50-57, Jan. 2006.

[44] Ö. Ergül and L. Gürel, "The use of curl-conforming basis functions for the magnetic-field integral equation," IEEE Trans. Antennas Propag., vol. 54, no. 7, pp. 1917-1926, Jul. 2006.

[45] L. C. Trintinalia and H. Ling, "First order triangular patch basis functions for electromagnetic scattering analysis," J. Electromagn. Waves and Appl., vol. 15, no. 11, pp. 1521-1537, 2001.

[46] Ö. Ergül and L. Gürel, "Improving the accuracy of the magnetic field integral equation with the linear-linear basis functions," Radio Sci., vol. 41, Jul. 2006, RS4004, doi:10.1029/2005RS003307.

[47] Ö. Ergül and L. Gürel, "Linear-linear basis functions for MLFMA solutions of magnetic-field and combined-field integral equations," IEEE Trans. Antennas Propag., vol. 55, no. 4, pp. 1103-1110, Apr. 2007.

[48] C. P. Davis and K. F. Warnick, "High-order convergence with a low-order discretization of the 2-D MFIE," IEEE Antennas Wireless Propag. Lett., vol. 3, pp. 355-358, 2004. 


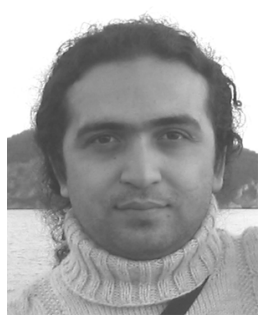

Özgür Ergül (S'98) received B.Sc. and M.S. degrees in electrical and electronics engineering from Bilkent University, Ankara, Turkey, in 2001 and 2003, respectively. He is currently pursuing the Ph.D. degree at Bilkent University.

Since 2001, he has served as a Teaching and Research Assistant in the Department of Electrical and Electronics Engineering at Bilkent University. He has been affiliated with the Computational Electromagnetics Group at Bilkent University from 2000 to 2005 and with the Computational Electromagnetics Research Center (BiLCEM) since 2005. His research interests include fast and accurate algorithms for the solution of electromagnetics problems involving large and complicated structures, integral equations, parallel programming, and iterative techniques.

Mr. Ergül is a recipient of the 2007 IEEE Antennas and Propagation Society Graduate Fellowship and the 2007 Leopold B. Felsen Award for Excellence in Electrodynamics. He is the Secretary of Commission E (Electromagnetic Noise and Interference) of URSI Turkey National Committee. His academic endeavors are supported by the Scientific and Technical Research Council of Turkey (TUBITAK) through a Ph.D. scholarship.

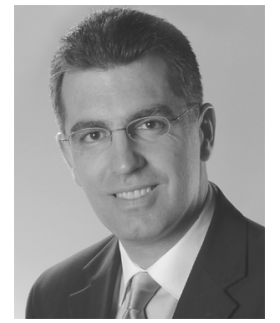

Levent Gürel (S'87-M'92-SM'97-F'09) received the B.Sc. degree from the Middle East Technical University (METU), Ankara, Turkey, in 1986 and the M.S. and Ph.D. degrees from the University of Illinois at Urbana-Champaign (UIUC), in 1988 and 1991, respectively, all in electrical engineering.

He joined the Thomas J. Watson Research Center of the International Business Machines Corporation, Yorktown Heights, New York, in 1991, where he worked as a Research Staff Member on the electromagnetic compatibility (EMC) problems related to electronic packaging, on the use of microwave processes in the manufacturing and testing of electronic circuits, and on the development of fast solvers for interconnect modeling. Since 1994, he has been a faculty member in the Department of Electrical and Electronics Engineering of the Bilkent University, Ankara, where he is currently a Professor. He was a Visiting Associate Professor at the Center for Computational Electromagnetics (CCEM) of the UIUC for one semester in 1997. He returned to the UIUC as a Visiting Professor in 2003-2005, and as an Adjunct Professor after 2005. He founded the Computational Electromagnetics Research Center (BiLCEM) at Bilkent University in 2005, where he is serving as the Director. His research interests include the development of fast algorithms for computational electromagnetics (CEM) and the application thereof to scattering and radiation problems involving large and complicated scatterers, antennas and radars, frequency-selective surfaces, high-speed electronic circuits, optical and imaging systems, nanostructures, and metamaterials. He is also interested in the theoretical and computational aspects of electromagnetic compatibility and interference analyses. Ground penetrating radars and other subsurface scattering applications are also among his research interests. Since 2006, his research group has been breaking several world records by solving extremely large integral-equation problems, most recently the largest involving as many as 205 million unknowns.

Prof. Gürel is a member of the General Assembly of the European Microwave Association (EuMA), a member of the USNC of the International Union of Radio Science (URSI), and the Chairman of Commission E (Electromagnetic Noise and Interference) of URSI Turkey National Committee. His many awards and accomplishments include two notable awards from the Turkish Academy of Sciences (TUBA) in 2002 and the Scientific and Technical Research Council of Turkey (TUBITAK) in 2003. He served as the Chairman of the AP/MTT/ED/EMC Chapter of the IEEE Turkey Section in 2000-2003. He founded the IEEE EMC Chapter in Turkey in 2000. He served as the Cochairman of the 2003 IEEE International Symposium on Electromagnetic Compatibility. He organized and served as the General Chair of the CEM'07 Computational Electromagnetics International Workshop in 2007. He is currently serving as an associate editor for Radio Science, IEEE Antennas and Wireless Propagation Letters, Journal of Electromagnetic Waves and Applications, and PIER. 